\title{
On Using Physical Analogies for Feature and Shape Extraction in Computer Vision
}

\author{
Mark S. Nixon, Xin U. Liu, Cem Direkoğlu and David J. Hurley \\ School of Electronics and Computer Science, University of Southampton UK \\ $\{$ msn|cd05r|x104r\}@ecs.soton.ac.uk and david@hurley25.fsnet.co.uk
}

\begin{abstract}
There is a rich literature of approaches to image feature extraction in computer vision. Many sophisticated approaches exist for low- and high-level feature extraction but can be complex to implement with parameter choice guided by experimentation, but impeded by speed of computation. We have developed new ways to extract features based on notional use of physical paradigms, with parameterisation that is more familiar to a scientifically-trained user, aiming to make best use of computational resource. We describe how analogies based on gravitational force can be used for low-level analysis, whilst analogies of water flow and heat can be deployed to achieve high-level smooth shape detection. These new approaches to arbitrary shape extraction are compared with standard state-of-art approaches by curve evolution. There is no comparator operator to our use of gravitational force. We also aim to show that the implementation is consistent with the original motivations for these techniques and so contend that the exploration of physical paradigms offers a promising new avenue for new approaches to feature extraction in computer vision.
\end{abstract}

Keywords: Feature extraction, Shape detection, Image processing, Computer vision, Force field, Water flow, Heat

\section{FEATURE EXTRACTION AND IMAGE PROCESSING}

\subsection{Feature and Shape Extraction}

There is now a rich literature of techniques which can detect low-level features, such as edges and corners, and high level shapes [1]. Low-level operators are generally those which operate on an image as a whole; high level operators are those which process images so as to determine shapes that lie therein. Both processes are used within computer vision, to render explicit information that is implicit within the original image - as such providing image understanding.

The state-of art operators for low-level feature extraction include anisotropic diffusion for image smoothing, to preserve features and to reduce the effects of noise; the Scale Invariant Feature Transform (SIFT) aims to find corner features that persist over image scales, by a sophisticated operation based on the Difference of Gaussian Operator. By way of example, we show the result of anisotropic diffusion [2] applied to an image of an eye, in comparison with the result by Gaussian filtering, a standard operator. The anisotropic diffusion process in Figure 1(c) achieves a more pleasing result than that of Figure 1(b), preserving features better whilst smoothing noise to greater effect. The parameter choice for the Gaussian operator is window size and the variance of the Gaussian operator; the parameter choice for anisotropic diffusion largely revolves around two main parameters of which $\lambda$ offers differing levels of (isotropic) smoothing, and $k$ controls the (anisotropic) feature preservation process (another parameter is the number of iterations and this can be few in practice). There is greater choice of functionals 
for edge preservation in anisotropic diffusion and we show here the basic process. The effects of alternative choice for the parameters are shown in Figures 1(d)-1(e); these are easily investigated since the operator is fast when implemented on a modern computer.

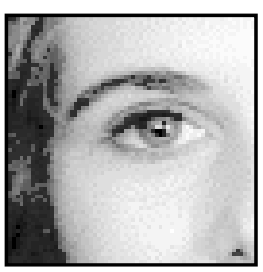

(a) original image

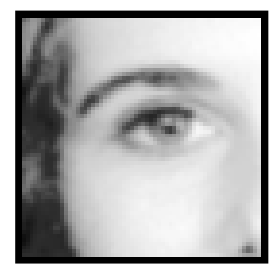

(b) result by Gauss-
ian smoothing

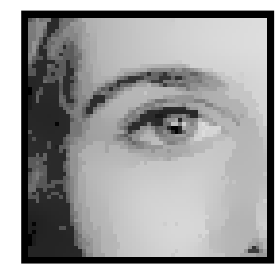

(c)-(e) results by anisotropic diffusion, and parameter

(c) $k=5 ; \lambda=0.25$ settings

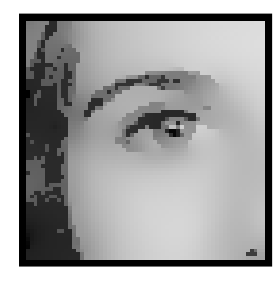

(d) $k=15$;

$\lambda=0.25$

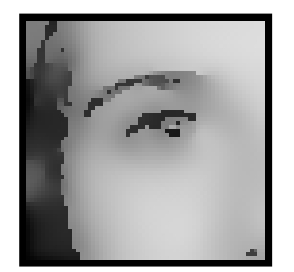

(e) $k=25$;

$\lambda=0.25$

FIGURE 1 Filtering by the Gaussian Operator and by Anisotropic Diffusion [1]

The range of techniques for high-level shape extraction includes techniques which operate by matching, such as template matching or the Hough transform, active contours and statistical shape models. To achieve a result by matching requires knowledge of the target shape. If this target is not yet known, then evolutionary techniques are used which match feature extraction to chosen image properties. Amongst the most popular operators for arbitrary shape extraction are geodesic active contours which evolve to find a target shape contour. Example results by curve evolution are shown in Fig. 2 [3]. These show that the technique can converge to an acceptable result, Fig. 2(f) - showing the palm of the hand and the fingers - though with an extra finger due to the background, from an initialisation which was a circle which did not wholly encompass the target feature, shown in Fig. 2(a). Overall the curve evolution techniques can achieve spectacular results, but this requires selection for a number of parameters, and this can be difficult to optimise given the enormous computations involved in rebalancing the level sets, used in the formulation, at each iteration of the technique. There have been many developments in the field and newer approaches use texture to guide segmentation [4].

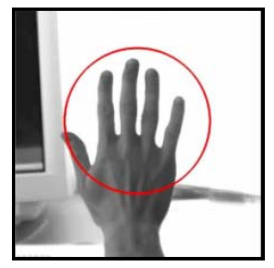

(a) initialisation

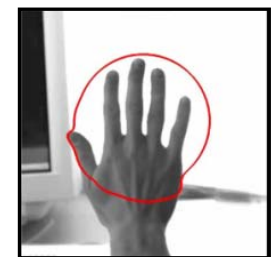

(b) iteration 1

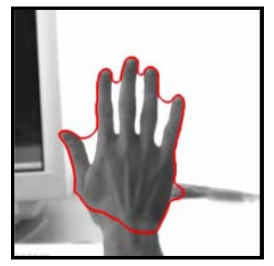

(c) continuing..

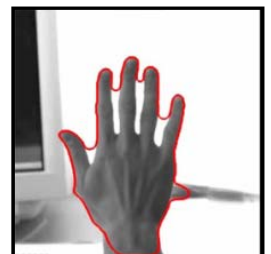

(d) continuing..

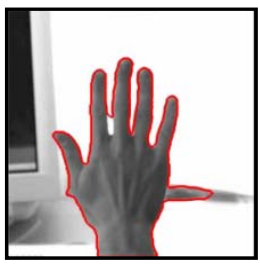

(f) final result

FIGURE 2 Extraction by curve evolution (a diffusion snake) [3]

Naturally, performance depends on parameter selection. A major advantage concerns initialisation, as the curve evolution techniques evolve to determine a solution within the image which is consistent with desired properties. The advantage is that the initialisation can be within or outside the target shape (though this is not a consistent advantage when initialisation within a shape can be guaranteed).

By way of illustration, we shall use the technique which many people compare the result of their own new approach with is a geometric active contour called the active contour without edges, introduced by Chan and Vese [5]. Their model uses regional statistics for segmentation, and as such is a region-based level set model. The overall premise is to avoid using gradient (edge) information since this can lead to boundary leakage and cause the contour to collapse. The active contour without edges model can address problems with initialization, noise and boundary leakage (since it uses regions, not gradients) but still suffers from computational inefficiency and difficulty in implementation, because of the level set method. An example result is shown in Fig. 3 where the target aim is to extract outside boundary of the hippopotamus the active contour without edges aims to split the image into the extracted object (the hippopotamus) and its background (the grass). In order to do this, we need to specify an 
initialisation which we shall choose to be within a small circle inside the hippopotamus, as shown in Fig. 3(a). The result of extraction is shown in Fig. 3(b) and we can see that the technique has detected much of the hippopotamus, and the result is encouraging but not perfect. The values used for the parameters here were: $\lambda_{1}=\lambda_{2}=1.0 ; \eta=0$ (i.e., area was not used to control evolution); $y=0.1 \times 255^{2}$ (the length parameter was controlled according to the image resolution) and some internal parameters were $h=1$ (a 1 pixel step space); $\Delta t=0.1$ (a small time spacing) and $\varepsilon=1$ (a parameter within the step, and hence the impulse functions which calculation is based). Alternative choices are possible, and can affect the result achieved. The result here has been selected to show performance attributes; the earlier result (Fig. 2) was selected to demonstrate finesse.

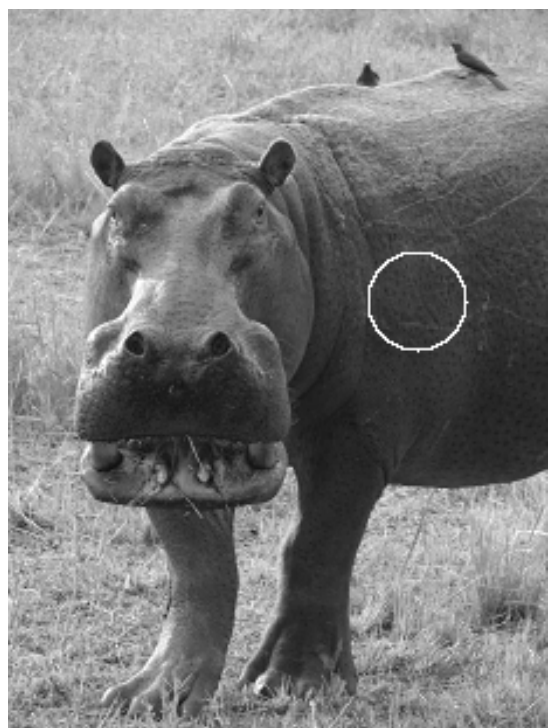

(a) initialisation

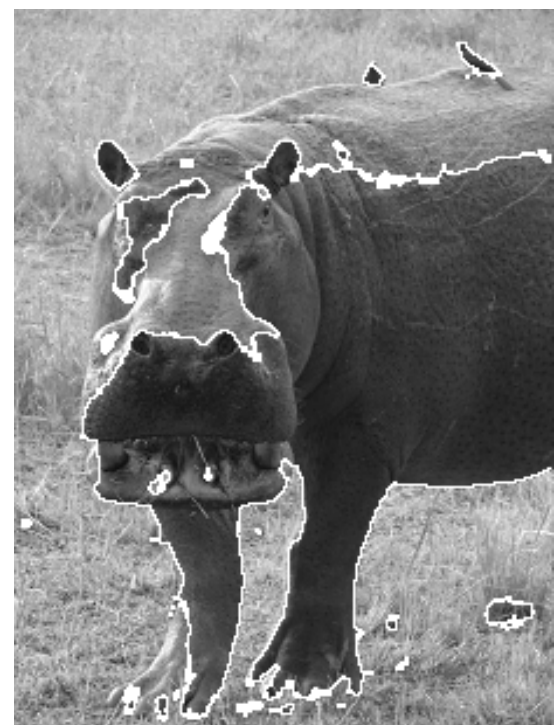

(b) result

FIGURE 3 Extraction by a Level-Set Based Approach [1]

As ever, there is no panacea in engineering and we should not expect one. The advance of new techniques often stimulates the development of new approaches. The developments in curve evolution have led to new approaches for shape extraction guided by image statistics. We seek here to use the image statistics in a new way, guided by use of physical analogies. The deployment is most certainly by analogy since images are simply collections of twodimensionally indexed values which are usually represented by brightness. It is in these collections of points we seek to determine shapes.

\subsection{On Using Physical Analogies for Feature Extraction}

Using physical analogies is a new basis for feature extraction which has as yet attracted scant attention. We describe some related approaches and later will compare results with established approaches with the same aim. We confine our analysis to those approaches which are directly related to our use of physical analogies, but note there have been other (minor) uses of physical analogies in other approaches, which are not mentioned here.

\subsubsection{Using Force Analogies in Image Processing}

Though there is no other work similar to our approach using a gravitational analogy, there are other force-based approaches motivating image processing feature extraction operators. In [6], magnetostatic theory was combined with an active contour model. For a moving charge inside a magnetic field, there are magnetic forces introduced by the field acting on it. Further, since currents are essentially sets of moving charges, there are magnetic forces acting on the currents inside magnetic fields. The object boundary generates a magnetic field and thus exerts magnetic forces upon the deformable contour driving it to the boundary position. The Biôt- 
Savart law defines the magnetic flux density generated by a conductor carrying a current at any pixel with position vector. The current direction is estimated by the signed distance transform, which is also used to initialise level set functions, as used in modern approaches to curve evolution. Therefore, the magnetic force can be easily combined with level sets. Electrostatic field theory has also been adopted in image segmentation. Jalba et al. [7] presented a charged particle model that simulates the particle movements in an electrostatic field. A set of positively charged free particles is positioned in the image analogical field distributed with fixed negative charges proportional to the edge strength. Because the free particles have opposite polarity to the fixed charges and the same polarity to each other, there are attractive forces (the Lorentz force) from the fixed charges to the free particles, and repellent forces (the Coulomb force) between the free particles.

\subsubsection{Deployment of Water and Heat in Image Analysis}

The most similar approaches to using water and heat as analogies for feature extraction is to deploy region growing. This is a feature extraction procedure that groups pixels or sub-regions to make larger regions, based on predefined similarity criteria for region growth. The basic approach starts with a seed point and merges neighbouring pixels that have pre-defined properties similar to the seed, such as intensity or texture. Although, region growing techniques can detect multiple objects simultaneously and can be more efficient than active contour or curve evolution models, the main problem is the appropriate selection of the similarity criteria. Region growing approaches also have to use connectivity information to define the neighbouring pixels in each step of growth. In addition, they can achieve region segmentation with irregular boundaries and holes in the presence of high noise, since they omit smoothing.

The most familiar use of water flow segmentation is floodfill, but this suffers in performance by not including water properties. Instead of model-based methods, some developed the morphological watershed based region growing techniques [8,9]. The approach is based on the fact that smooth surfaces can be decomposed into hills and valleys by studying critical points and their gradient. Considering pixel properties (intensity or gradient) as elevation and then simulating rainfall on the landscape, rain water will flow from areas of high altitude along lines of steepest descent to arrive at some regional minimal height. The catchment basins are defined as the draining areas of its regional minima and their boundaries can then be used in object extraction. Though assuming water collection, the method does not use the features of water itself and focuses on the image's geographical features. The non-linearity arising from issues like finding steepest descent lines between two points makes the method complicated. Moreover, the region growing framework often yields irregular boundaries, over-segmentation and small holes.

One of the most popular uses of heat in image processing is anisotropic diffusion which is a state-of-art process for image enhancement. In motion analysis, we can also see a significant application of heat flow by [10]. The algorithm combines anisotropic and isotropic heat flow to obtain moving edges. In [11], an anisotropic diffusion pyramid was introduced for region based segmentation. The pyramid is constructed using the scale space representation of the anisotropic diffusion. In [12], the anti-geometric heat flow model was introduced for the segmentation of regions. Here, anti-geometric heat flow is represented as diffusion through the normal direction of edges, for the extraction of smooth shapes.

\subsubsection{Contributions}

This paper is the first formal definition of the direction of approaches using physical analogies and the first unified description of a selection of new approaches to feature extraction, based on using analogies of force, water and heat. We show that parameterisation can be simplified, and using simpler computational frameworks, say than level sets. The parameter settings can also be interpreted more easily, in part since a developer can identify better with physical notions than can be achieved with recondite mathematical analysis, which often omits precise definition crucial to success. We describe our techniques in overview only, giving detailed references for alternative investigation. We outline results showing performance capability and though there are specific advantages for each technique, our usual approach is to demonstrate performance in noise, and in comparison where possible with a contemporaneous approach. 


\section{NEW PHYSICAL ANALOGIES FOR FEATURE EXTRACTION}

We describe a selection of new approaches, aimed to provide new techniques which allow insight for parameter selection, fast implementation by virtue of simplicity or by use of established techniques to improve computational speed, and with performance at least comparable with that achieved by state-or-art techniques for feature extraction in image processing and computer vision.

\subsection{Force Field Transform}

Hurley developed a transform called the force field transform $[13,14]$ which uses an analogy to gravitational force wherein mass is equivalent to pixel brightness and distance is measured between pixels. The transform pretends that each pixel exerts a force on its neighbours which is inversely proportional to the square of the distance between them. This generates a force field where the net force at each point is the aggregate of the forces exerted by all the other pixels on a "unit test pixel" at that point. This very large scale summation affords very powerful averaging which reduces the effect of noise. The approach was developed in the context of ear biometrics, recognising people by their ears, which has unique advantage as a biometric in that the shape of people's ears does not change with age, and of course - unlike a face - ears do not smile. The force field transform of an ear, Figure 4(a), is shown in Figure 4(b). Here, the averaging process is reflected in the reduction of the effects of hair. The transform itself has highlighted ear structures, especially the top of the ear and the lower 'keyhole' (the notch). As such, the features pertinent to recognition are preserved, and those which detract from recognition are reduced.

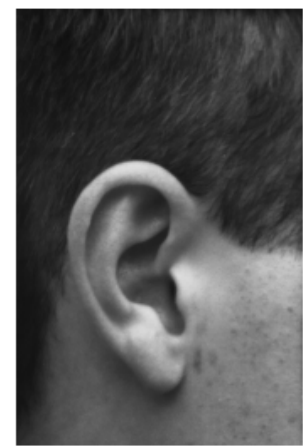

(a) image of an ear

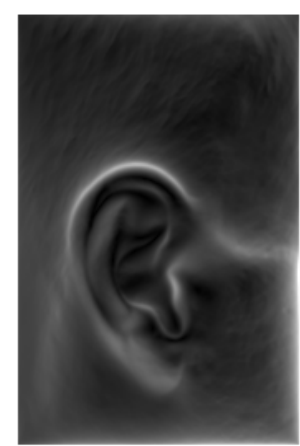

(b) magnitude of force field transform

FIGURE 4 Illustrating the Force Field Transform Results

The image shown is actually the magnitude of the force field. The transform itself is a vector operation, and includes direction [13] which can be exploited in later feature extraction. The transform is expressed as the calculation of the force $\mathbf{F}$ between two points at positions $\mathbf{r}_{i}$ and $\mathbf{r}_{j}$ which is dependent on the value of a pixel at point $\mathbf{r}_{i}$ as

$$
\mathbf{F}_{i}\left(\mathbf{r}_{j}\right)=\mathbf{P}\left(\mathbf{r}_{i}\right) \frac{\mathbf{r}_{i}-\mathbf{r}_{j}}{\left|\mathbf{r}_{i}-\mathbf{r}_{j}\right|^{3}}
$$

given brightness $\mathbf{P}$ at point $\mathbf{r}_{i}$ and which assumes that the point $\mathbf{r}_{j}$ is of unit "mass". This is a force (and thus directional, which is why the inverse square law is expressed as the ratio of the difference to its magnitude cubed) and the magnitude and directional information has been exploited to determine an ear 'signature' by which people can be recognised.

An example for the calculation of the force field at a point is given in Figure 5, with vector lengths indicating magnitude and so showing the resulting force F. In application, Equation 1 can be used to define the coefficients of a template that is convolved with an image (implemented by the Fast Fourier Transform to improve speed). Note that this transform actually exposes low level features (the boundaries of the ears) which is also the focus of edge detection. As such, noise has been reduced in the hair whilst low-level features have been exposed, by a technique which does not require any parameter choice, unlike the results achieved by anisotropic diffusion. 


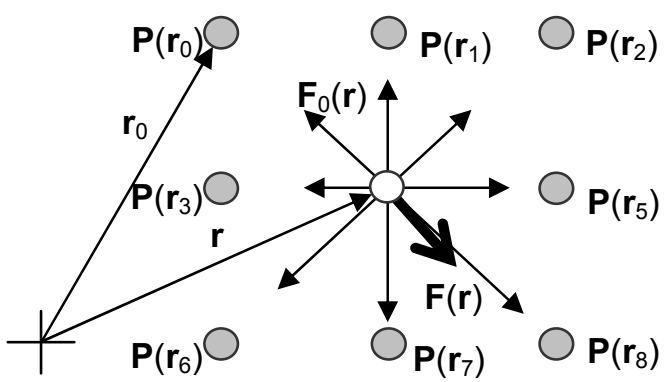

FIGURE 5 Force Field Calculation at a Single Pixel Position

To find the total potential energy at a particular pixel location in the image, the scalar sum is taken (over the $N$ image points) of the values of the overlapping potential energy functions of all the image pixels at that precise location as

$$
E\left(\mathbf{r}_{j}\right)=\sum_{i \in 0, N-1 \mid i \neq j} E_{i}\left(\mathbf{r}_{j}\right)=\sum_{i \in 0, N-1 \mid i \neq j} \frac{\mathbf{P}\left(\mathbf{r}_{i}\right)}{\left|\mathbf{r}_{i}-\mathbf{r}_{j}\right|}
$$

The invariant advantages are that the (force and energy) transform does not change with addition of mean-zero noise as shown in Eq. 3 where $v_{j}$ represents the noise affecting point $\mathbf{r}_{\mathrm{j}}$, here shown for the energy as

$$
\begin{aligned}
E\left(\mathbf{r}_{j}\right)=\sum_{i \in 0, N-1 \mid i \neq j} E_{i}\left(\mathbf{r}_{j}\right)= & \sum_{i \in 0, N-1 \mid i \neq j} \frac{\mathbf{P}\left(\mathbf{r}_{i}\right)+v_{j}}{\left|\mathbf{r}_{i}-\mathbf{r}_{j}\right|} \\
& =\sum_{i \in 0, N-1 \mid i \neq j} \frac{\mathbf{P}\left(\mathbf{r}_{i}\right)}{\left|\mathbf{r}_{i}-\mathbf{r}_{j}\right|}+\sum_{i \in 0, N-1 \mid i \neq j} \frac{v_{j}}{\left|\mathbf{r}_{i}-\mathbf{r}_{j}\right|}
\end{aligned}
$$

and with noise of mean-zero Gaussian distribution the latter term is mean-zero, thus returning the energy to the form of Eq. 2. Naturally, localised variation in image brightness will change the overall shape of the force field, but not its nature, and for scaling brightness by a chosen factor $k$ will change the energy as

$$
\begin{aligned}
E\left(\mathbf{r}_{j}\right)=\sum_{i \in 0, N-1 \mid i \neq j} E_{i}\left(\mathbf{r}_{j}\right) & =\sum_{i \in 0, N-1 \mid i \neq j} \frac{k \mathbf{P}\left(\mathbf{r}_{j}\right)}{\left|\mathbf{r}_{i}-\mathbf{r}_{j}\right|} \\
& =k \sum_{i \in 0, N-1 \mid i \neq j} \frac{\mathbf{P}\left(\mathbf{r}_{j}\right)}{\left|\mathbf{r}_{i}-\mathbf{r}_{j}\right|}
\end{aligned}
$$

and so will affect the force field in proportion by changing its scale, rather than its shape. These properties led to its efficacious use as a pre-processor prior to feature extraction for ear biometrics [13]. This was achieved by determining minima within the force field functional: the relative positions of the minima were the same for the same person (and invariant to brightness scaling, initialisation and position) and the set of minima were different between different people thus allowing the force field transform to be used as a precursor to biometric deployment. The positions of the minima are shown for four different people in Fig. 6 where the image on the left shows the positions of the force field minima (highlighted in white) and the image on the right shows the evolution which led to determining these positions.

A further advantage of the force field approach is that it allows for development of differential vector analysis, leading to a new approach to measure curvature [14] and hence leading to an extended approach to biometrics. 

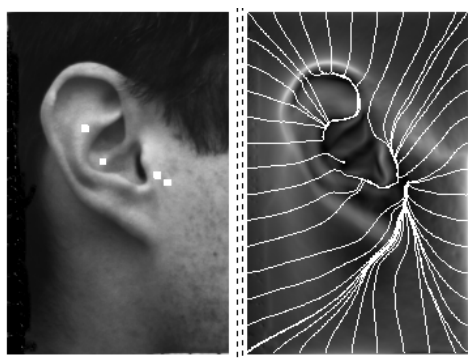

(a) Ear 1

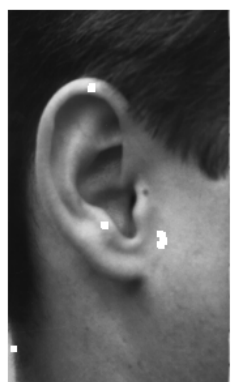

(c) Ear 3
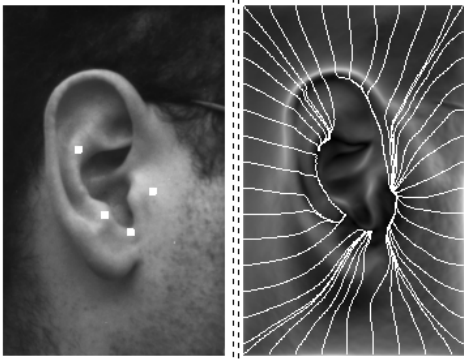

(b) Ear 2

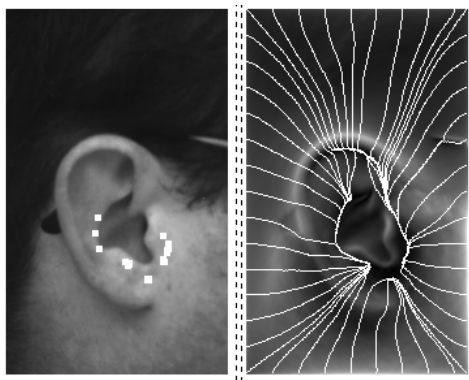

(d) Ear 4

FIGURE 6 Uniqueness of Ears by Force Field Transform Analysis

\subsection{Feature Extraction by Water Flow}

\subsubsection{Approach}

In developing our approach based on water flow to find arbitrary shapes by evolution, we did not seek to use the sophisticated (though complex) finite element models since we specifically aimed for low computational complexity. Water flow is a compromise between several factors: the position of the leading front of a water flow depends on pressure, surface tension, and adhesion/capillarity. There are some other natural properties like turbulence and viscosity, which are ignored here. Image edges and some other characteristics that can be used to distinguish objects are treated as the "walls" terminating the flow. The final static shape of the water should describe the related object's contour. The flow is determined by pressure and the resistance; the relationship between the flow rate $f_{r}$, the flow resistance $R$ and the pressure difference, is given by:

$$
f_{r}=\frac{P_{i}-P_{o}}{R}
$$

where $P_{i}$ and $P_{o}$ are pressure of the inflow and outflow, respectively. The pressure difference drives the flow and

$$
f_{r}=A \cdot V_{\text {effective }}
$$

where $A$ is the cross-sectional area and $V_{\text {effective }}$ is the effective flow velocity. Hence the velocity can be related to force and resistance through Eqns. 5 and 6 .

These can be used as a basis for simulating water flow in images. Given an initialisation point, we need to determine where water will flow, according to selected image forces. The basis of our approach uses pressure differential Eqn. 5 moderated by surface tension and adhesion [15, 16]. The water element can move outwards in any direction for which the component of velocity is positive. However, only if the velocity in the direction is sufficiently large, can the element break through the image resistant forces and reach the new position. To reconcile the flow velocity with forces, dynamical formulae are used. We may compute the displacement of a contour element on each possible direction within a fixed time interval, which is similar to snake techniques. However, for simplicity and avoiding the interpolation problem, a framework like region growing and the greedy snake is used: the element will flow to some positions if certain conditions or formulae are satisfied. Here, an equation describing the 
conservation of energy is employed. If assuming that an element, which has a positive velocity $v$ on a particular direction and is acted by the force $F$ during the process, can arrive at the direction-related position ultimately, then this equality must be fulfilled:

$$
m v_{F}^{2} / 2=F S+m v^{2} / 2
$$

where $v_{F}$ is the final scalar velocity after fixed displacement $S$ and $m$ is the assumed mass. In this equation, force $F$ is a scalar which is positive when the force is consistent with velocity $v$, and negative otherwise. The summation on the right hand side is just the movement decision operator: only if $F$ is negative, can the summation be negative and thus the equality above cannot be satisfied.

Equation 7 provides the inequality to determine the feasibility of outwards flow. For each contour element, we have equations computing driving force $F_{\mathrm{D}}$ modified by surface tension, adhesion $F_{\mathrm{A}}$ and resistance $R$. There are other factors of water flow that could be included (such as turbulence) but these are not necessary at this stage. The flow velocity $\mathrm{V}$ can then be obtained through Eqn. 6 . If the velocity points towards the exterior of the water, the element is assumed to leave the original position. A unified image force $F$ is then computed. The result from the summation in Eqn. 7 determines the result of the movement.

Defining $m$ and $S$ in Eqn. 7 as constants, we can then present the new and detailed expression with parameters defined before:

$$
J=\Lambda\left(\left(F_{D}+F_{A}\right) / R(x, y)\right)^{2}+F
$$

Where $R$ is the edge response at point $(x, y), \Lambda$ is a regularization parameter set by users which controls the tradeoff between the two energy terms. It can be considered to be determined by the combination of mass $m$, displacement $S$ and area $A$. Its value reflects smoothing of image noise. For example, more noise requires larger value to be selected for $\Lambda$. $F_{A}$ and $F_{D}$ are the scalar components on the movement direction of $F_{\mathrm{A}}$ and $F_{\mathrm{D}}$, respectively. A positive direction is defined from the origin to the target. The movement decision can be completely made by this operator since the term of right hand side inside the brackets gives the velocity information and $J$ corresponds to the ultimate kinetic energy. If the velocity component is greater than zero and if $J$ is positive, the movement is said to be feasible and the target point will be flooded by water.

\subsubsection{Example Results}

Natural images with complex topology are also assessed. Figure 7 shows the result for the image of a river delta with different parameters, where the outer perimeter of the river is the target object. It is suited to performance evaluation since gaps and "weak" edges exist in the image. One example is the upper part of the river, where boundaries are blurred and irregular. There are also inhomogeneous areas inside the river, which are small islands and have lower intensity. Our water flow based operator can overcome these problems. As shown in Figure 7(a) by the dark line, a reasonably accurate and detailed contour of the river is extracted. At the upper area, some very weak boundaries are also detected. This is achieved by using high value of $k$ that gives the operator a high sensitivity to edge response. The contour is relatively smooth by virtue of surface tension. The fluidity leading to topological adaptability is shown well by successful flow to the branches at the lower area. Most of them are detected except failure at several narrow branches. The barriers are caused either by natural irregularities inside them or noise. In contrast, a lower value for $k$ relaxes the influence of strong edges, so the water flows to edges with weaker contrast, as shown by the bright boundary in Figure 7(b).

Chan's region-based level set approach [5] has also been applied to this image for comparison, as shown by Figure 7(c-d). Of the four main parameters, the length $\gamma$ exerts most significant influence to the segmentation results - if $y$ is small, the level set operator will detect as many details as possible; if $y$ is larger, the smaller points will not be detected and instead more general shape of the objects of interest will be segmented. Figure 7(c) shows the result with small $y$ where the final contours are the brighter areas (and there are many of them), where all the narrow branches of the river have been detected, as well many unwanted small shapes. The result in the upper area of the river is also corrupted by the over-segmentation. In Figure 
$7(d)$, we can see that a very large $y$ detects the general shape of the river basin (again, the brighter areas) and reduces the over-segmentation problem significantly (though still more severe than the water flow result in Figure $7(b))$. Nevertheless, the left upper part of the river basin is not detected at all by the level set operator.
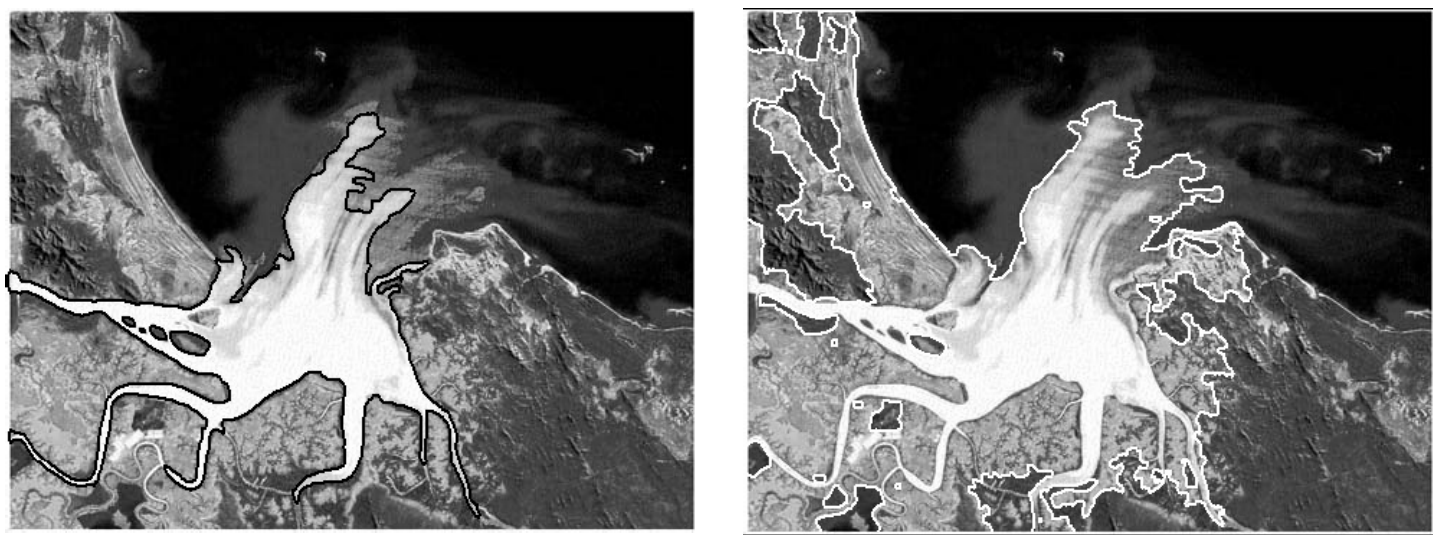

$a$ and $b$ Extraction by Water Flow

(a) bounding flow by strong edges $(\alpha=0.7$, $\Lambda=3, k=5)$

(b) bounding flow by weak edges $(\alpha=0.5, \Lambda$
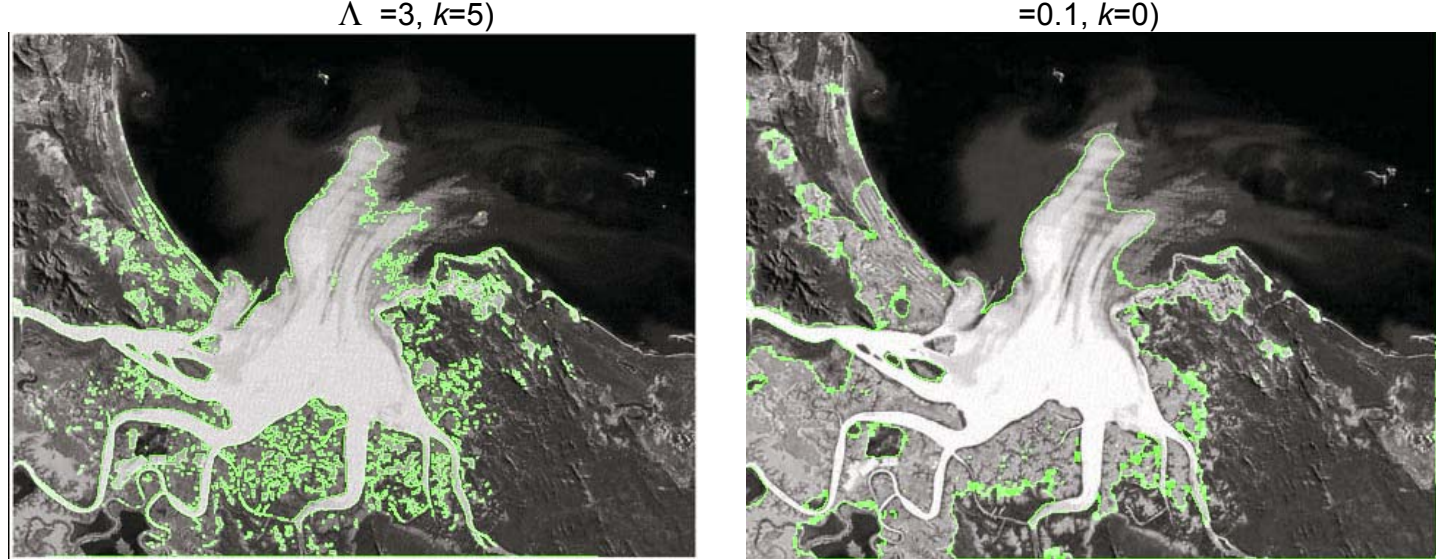

C and d Extraction by Curve Evolution

(c) $\lambda_{1}=\lambda_{2}=1, \eta=0, \gamma=0.01^{*} 255^{2}$

(d) $\lambda_{1}=\lambda_{2}=1, \eta=0, y=0.2^{*} 255^{2}$

FIGURE 7 Extracting Natural Features by Water Flow

The immunity to noise is also assessed quantitatively, in comparison with a level set approach [5], and Figure 8 shows the result. The Mean Square Error is used as the criterion with a synthetic test image as the ground truth, which has been deliberately designed to incorporate a narrow boundary concavity. The remainder of the target feature is a circle, so the MSE is that computed for the difference of the best fit circle by the Hough Transform (HT), compared with the original circle parameters (the HT being chosen for provision of this metric by its ability to deliver a result equivalent to matched filtering). An example of the detection result is also shown for both approaches. Clearly, the water-flow approach has a better ability in high levels of noise (when the Signal to Noise ratio is low), since the MSE of the water flow is much lower than for the level set approach; when the noise is much smaller, both approaches can produce very accurate results. The smoothness of the water-flow approach (like the viscosity of the water) means that the extraneous details in the level set approach are not found by waterflow, leading to a better result. The level set approach is much slower when both are implemented in Matlab. 


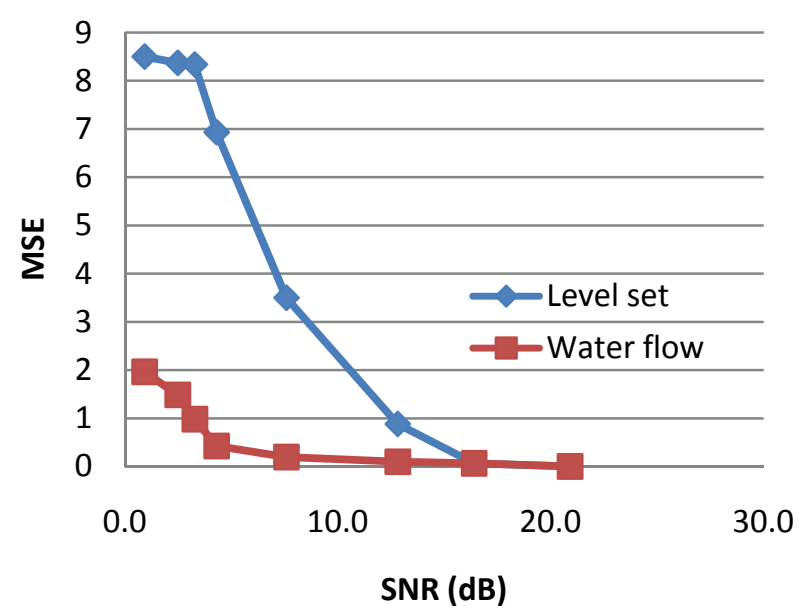

(a) MSE of circle parameters for differing levels of impulsive and Gaussian noise

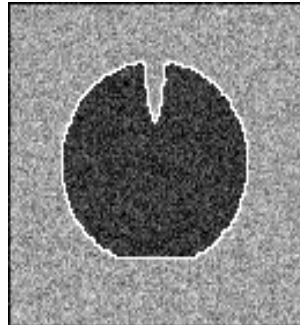

(b) example water flow result (white)

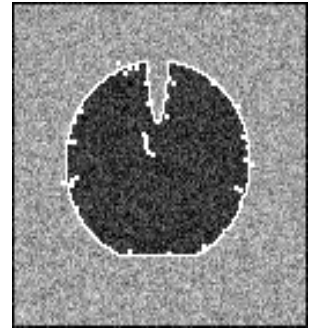

(c) example level set result (white)

FIGURE 8 Extracting Circles in Noisy Images

The 3-D water flow model is expected to have comparative performance in volume segmentation. We have applied our 3-D water model to a variety of medical images so as to segment anatomical structures with complex shapes and topologies [16]. Figure 9 presents a typical example where the model is applied to a $181 \times 217 \times 181 \mathrm{MR}$ image volume of a human brain. The water source is set inside the lateral ventricles and the parameters are set at $k=5$, $\alpha=0.5, \Lambda=1$. The operator detects most parts of the lateral ventricles. Two cross-sections of the fitted model in upper and lower slices are also shown in Figure 9.

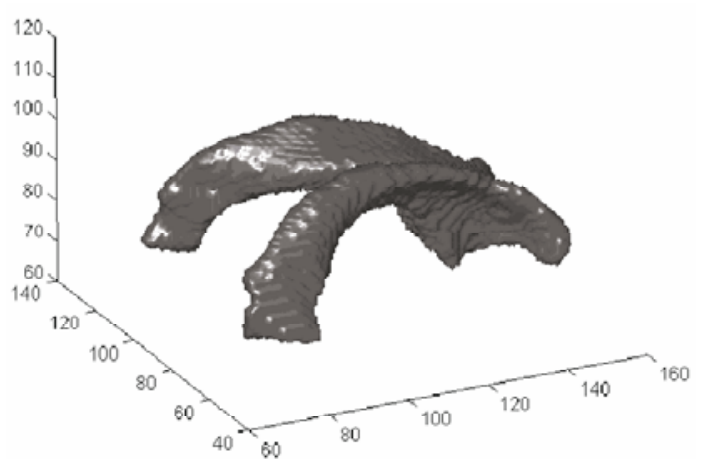

(a) water flow segmentation of the lateral ventricles

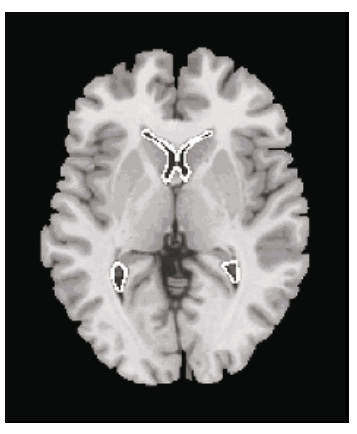

(b) lower cross-section

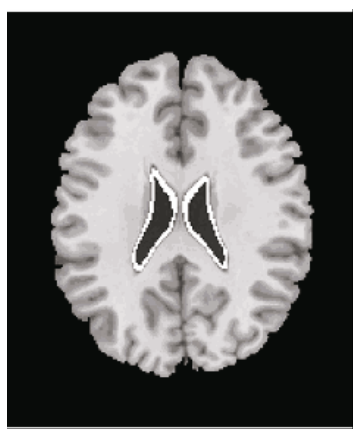

(c) upper cross-section

FIGURE 9 An example of the MRI volume segmentation by 3-D water flow analogy

\subsection{Using Heat for Feature Extraction}

\subsubsection{Approach}

Conduction, convection and radiation are three different modes of heat flow. Here, we chose to investigate use of a conduction model, which we found to operate well. Conduction is the flow of heat energy from high- to low- temperature regions due to the presence of a thermal gradient in a body. The change of temperature over time at each point of material is described using the general heat conduction or diffusion equation,

$$
d T / d t=\alpha\left(d^{2} T / d x^{2}+d^{2} T / d y^{2}\right)+Q=\alpha \nabla^{2} T+Q
$$


where, $\nabla$ represents gradient, $\alpha$ is called thermal diffusivity of the material and a larger values of $\alpha$ give faster heat diffusion through the material. $Q$ is the source term that applies internal heating. It can be uniformly or non-uniformly distributed over material body. The solution of this equation provides the temperature distribution over the material body and it depends on time, distance, heat source, properties of material, as well as specified initial and boundary conditions.

Consider a two-dimensional conductive solid body with initial and boundary conditions respectively given by $T(\mathbf{x}, t=0)=0$ and $T(\mathbf{x}, t)=0$, which mean the temperature is initially zero inside the body and the boundary condition is Dirichlet that has specified temperature (zero) at the boundaries. If we initialize a continuous heat source, which is a positive constant, at a point inside the body, there will be heat diffusion to the other points from the source position. As a result of this, all the points inside the body will have temperature values exceeding zero, except the boundary points. This is then an ideal approach for object segmentation in computer images. Let us investigate the proposed problem on a on a grey-level image. Assume that all the temperature values of the objects and the background are kept in another image, which is represented by $I$, and the initial condition of whole image is zero, $I(\mathbf{x}, t=0)=0$. This assumption means that all objects have temperature initially zero inside, as well as at the boundaries. When we initialize a heat source at any pixel inside the object, there will be heat diffusion to the other pixels from the source position, which will cause temperature to increase. However the temperature at the boundary layer must be kept at zero all the time to obtain the Dirichlet condition, where the boundary layer is defined at the external side of an object. To achieve this, we use a control function in the heat conduction equation as given below,

$$
d I(\mathbf{x}, t) / d t=C F(\mathbf{x}, t)\left(\alpha \nabla^{2} I(\mathbf{x}, t)\right)+Q(\mathbf{x})
$$

where $I(\mathbf{x}, t)$ represents an image pixel value in terms of temperature at each point and time, $\alpha$ is the thermal diffusivity and $0 \leq \alpha \leq 0.25$ for the numerical scheme to be stable in twodimensional system, $Q(\mathbf{x})$ is the source term and $\operatorname{CF}(\mathbf{x}, t)$ is the control function. The control function is obtained from the region statistics of source location on a given grey-level image. The proposed region statistics model is similar to an earlier one. Then, the following logical decision is applied in each position at each iteration

$$
C F(\mathbf{x}, t)=\left\{\begin{array}{cc}
1, & \sigma_{1}(\mathbf{x}, t) \leq \sigma_{2}(\mathbf{x}, t) \\
0, & \text { otherwise }
\end{array}\right.
$$

where $\sigma$ is the image variance and $\sigma_{1}$ is inside and $\sigma_{2}$ is outside the object. Therefore, the control function allows heat diffusion inside the object of interest and achieves the proposed Dirichlet condition on the boundary layer by keeping the temperature value at zero. However, it is better to start this process after a short diffusion time by assuming $C F(\mathbf{x}, t)=1$ at all points. Because, it will increase the number of samples inside of the TF, which means better decision at the first step especially for noisy cases. In addition, the heat source must be initialized onto a smooth surface of the object, since the source localization to the edge pixel will give the wrong region statistic for our purpose. Fig. 1(c) and (d) respectively show the evolution and the final position of the TF. However, there is no need to continue diffusion, after the TF reaches its final position. For this reason, the position of the TF is controlled at each iteration and when there is no movement, diffusion is terminated automatically.

The second stage of our approach is to use Geometric Heat Flow (GHF) which is a kind of anisotropic diffusion and is widely used for image denoising and enhancement. It diffuses along the boundaries of image features, but not across them. It derives its name from the fact that, under this flow, the feature boundaries of the image evolve in the normal direction in proportion to their curvature. Thus, GHF decreases the curvature of shapes while removing noise, in the images. Edge directions are related to the tangents of the feature boundaries of an image $B$. Let $\eta$ denote the direction normal to the feature boundary through a given point (the gradient direction), and let $\tau$ denote the tangent direction. Since $\eta$ and $\tau$ constitute orthogonal directions, the rotationally invariant Laplacian operator can be expressed as the sum of the 
second order spatial derivatives, $B_{\eta \eta}$ and $B_{\tau \tau}$, in these directions and the heat conduction equation can be written without using the source term,

$$
d B / d t=\alpha \nabla^{2} B=\alpha\left(B_{\eta \eta}+B_{\tau \tau}\right)
$$

Omitting the normal diffusion, while keeping the tangential diffusion yields the GHF equation as

$$
\frac{d B}{d t}=\alpha B_{\tau \tau}=\alpha \frac{\left(B_{x x} B_{y}^{2}-2 B_{x y} B_{x} B_{y}+B_{y y} B_{x}^{2}\right)}{\left(B_{x}^{2}+B_{y}^{2}\right)}
$$

In our model, GHF is used to decrease curvature for the purpose of obtaining smooth boundaries and removing holes that appear because of noise. This is achieved as follows. Firstly, a segmented region is converted to a binary form by thresholding (setting those points greater than zero to white).

\subsubsection{Example Results}

By way of example, we show the operation of this algorithm on noisy medical images, such as determining the ventricle in the $177 \times 178$ image of a human heart shown in Fig. 3(a) with the heat source location, we observe some drawbacks in segmentation. The drawbacks are irregular boundaries and holes inside the segmented region, as shown in Fig. 3(b). These problems are solved by using the heat flow analogy. In our model, GHF is used to decrease curvature for the purpose of obtaining smooth boundaries and removing holes that appear because of noise. This is achieved as follows. Firstly, a segmented region is converted to a binary form as given below and also shown in Fig. 3(c), the final segmentation is shown in Fig. $3(\mathrm{~d})$ and (e), where there are 50 iterations of the evolution process. Since the ventricle image seems bimodal, we can also consider the final form of the control function as shown in Fig. 3(f). To smooth boundaries and remove holes, we simply continue with Eq. (11) and observe the result in Fig. 3(g) and (h).

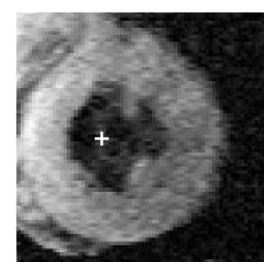

(a) Source position

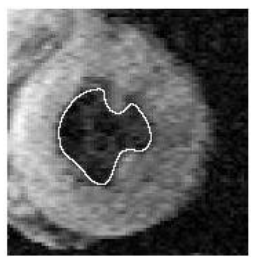

(e) Final shape after TF

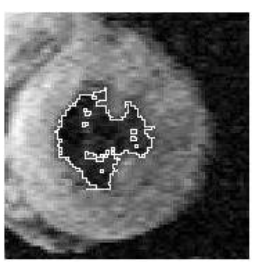

(b) Final TF at $t=59$

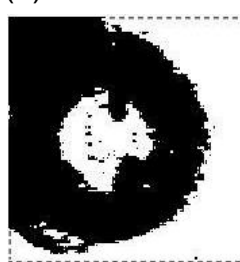

(f) $C F(\mathbf{x})$

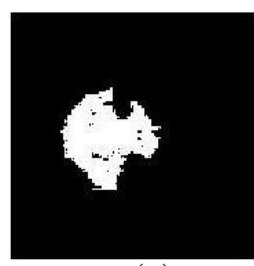

(c) $B(\mathbf{x})$

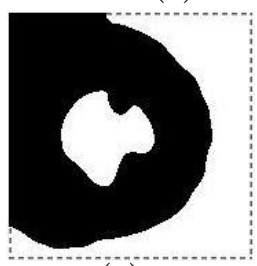

(g) $C F(\mathbf{x})$ after GHF

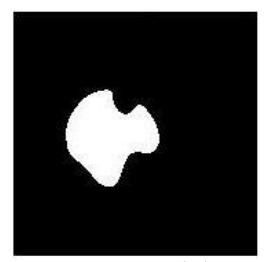

(d) $S(\mathbf{x})$

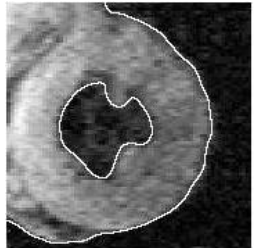

(h) Final shape

FIGURE 10 Illustration of heat flow for image segmentation. GHF is applied both to the binary form of the TF segmentation, $B(\mathbf{x})$, and to the control function $C F(\mathbf{x})$

Segmentation by TF is compared with the Active Contour Without Edges (ACWE) and Gradient Vector Flow Snake (GVFS). The evaluation is done on an irregular binary object with varying normal distributed noise $N\left(\mu, \sigma^{2}\right)$, as shown at the top row in Fig. 11. The sum of squared error (SSE) between the segmented and the original image (without noise) is employed to quantify the performance of each algorithm. In this evaluation, the contours and the heat source are initialized inside the harmonic object. Fig. 12 shows performance of TF, ACWE and GVFS in respect of increasing noise (as illustrated in Fig. 11). It is observed that TF and ACWE perform much better than GVFS. The reason for this is that TF and ACWE use region based algorithms, on the other hand GVFS uses a gradient based algorithm, which is very sensitive to the noisy conditions. When we compare TF and ACWE, ACWE performs better than TF until $\sigma \cong 40$. 

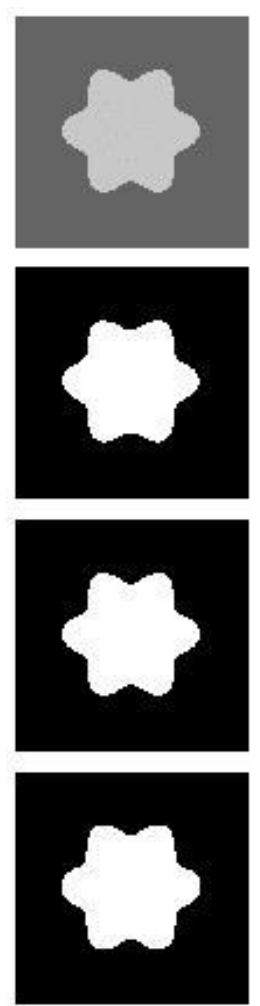

(a) $\sigma=0$
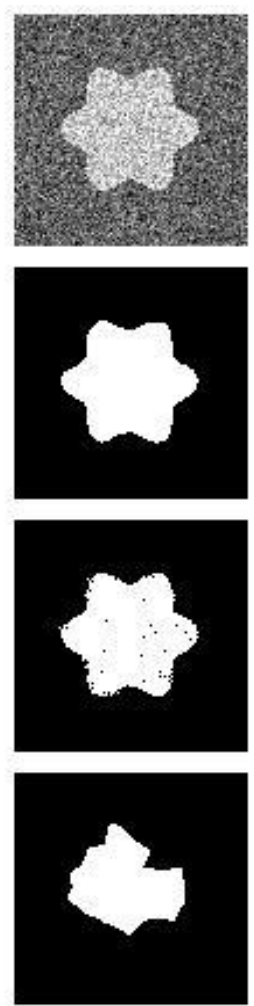

(b) $\sigma=40$
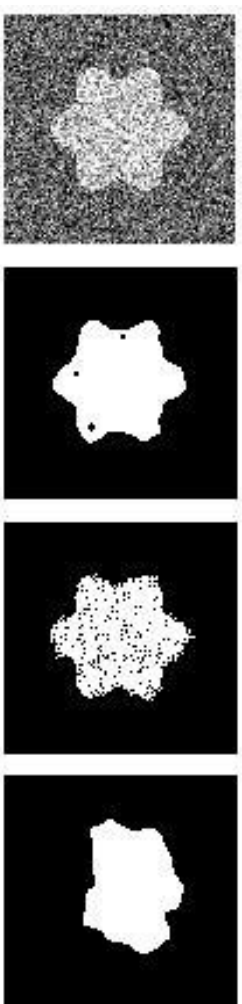

(c) $\sigma=60$
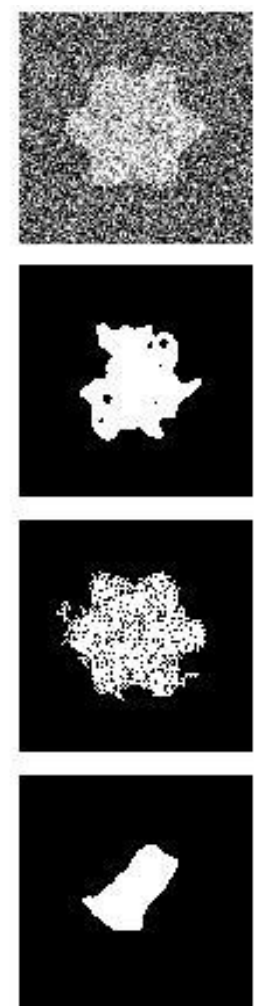

(d) $\sigma=80$
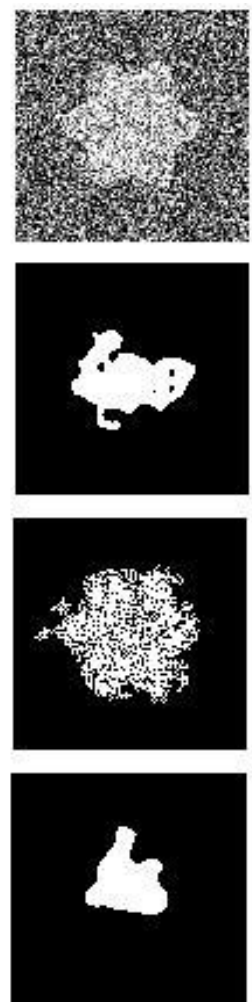

(e) $\sigma=100$

FIGURE 11 Results for TF (second row), ACWE (third row) and GVFS (fourth row) with respect to increasing Gaussian noise in the image of size $100 \times 100$.

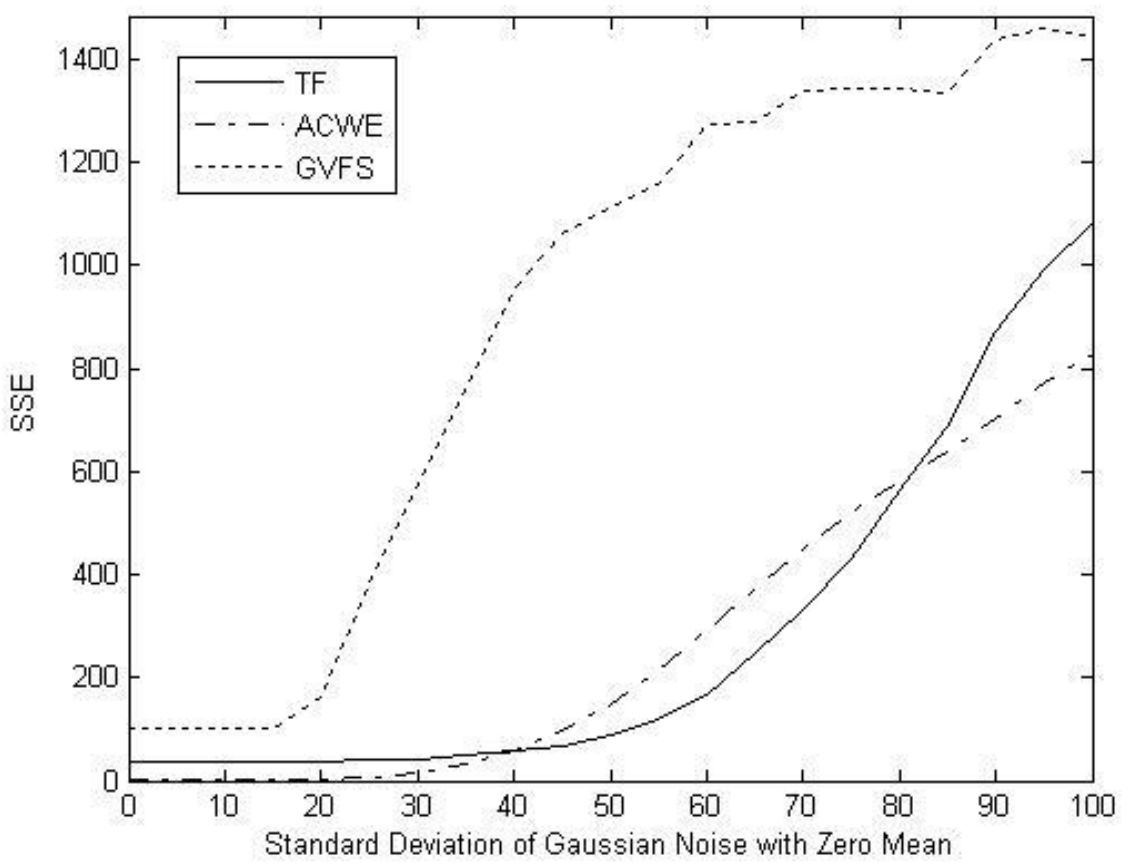

FIGURE 12 Performance of TF, ACWE and GVFS in increasing noise

This appears to be due to the smoothing operation in TF. TF segments better than ACWE. The main reason is again the smoothing operation. TF applies smoothing after rough segmentation without any relation to the regional statistic constraints, while ACWE uses smoothness constraint with regional statistic constraints during the segmentation. After $\sigma \cong 80$, it is seen that ACWE shows better performance than TF but all techniques start to fail in high noise, as 
expected - see Fig. 11(e). ACWE segments many regions outside the harmonic region in the presence of high noise and then some of the segmented noise remains connected to the original region when we select the biggest region.

Fig. 13 shows the segmentation of bimodal $123 \times 118$ human lung image by TF, CF and ACWE, where the initial contour for ACWE and the source position for TF are shown in Fig. 13(a)-(c), respectively, show the segmentation by TF and CF with the final result given by the white contour. Fig. 13(d) shows the segmentation by ACWE. All the parameters are same as in evaluation except the length parameter $y=0.08 \times 10^{2}$. All the evaluations and the simulation results are obtained by using MATLAB 7.0 on a Pentium IV computer, which runs Windows XP operating system with $3.2 \mathrm{GHz}$ CPU and 1GB RAM. It is observed that TF and CF achieves segmentation with $\mathrm{CPU}=1.96$ seconds and $\mathrm{ACWE}$ achieves with $\mathrm{CPU}=15.92$ minutes. This big difference in CPU time appears because of the computational complexity of ACWE that is implemented with level sets. It is also observed that CF can extract feature boundaries better than ACWE especially at the middle and at the bottom of the lung image. We have also extended the heat concept for feature extraction in images, via multiscale Fourier descriptions [18] (note that the original presentation by Fourier concerned heat).

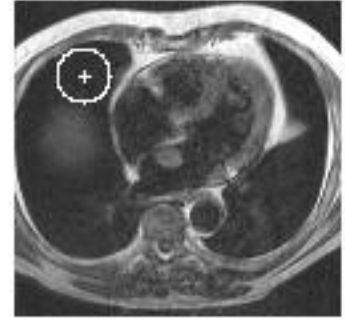

(a)

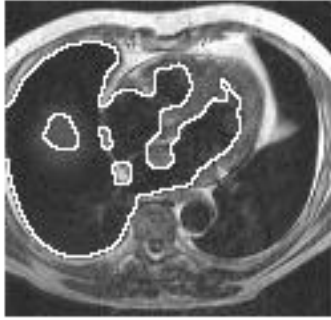

(b)

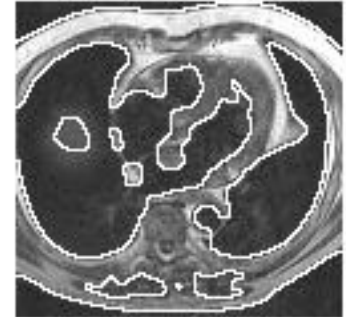

(c)

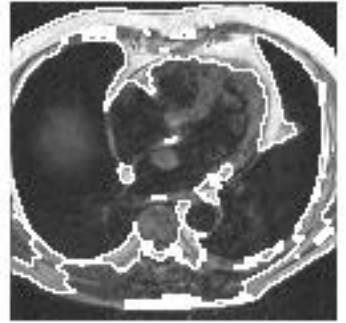

(d)

FIGURE 13 Segmentation of human lung image by TF, CF and ACWE. (a) Initial contour and the source position. (b) Segmentation by TF is shown with white contour on the image. (c) Segmentation by CF. (d) Segmentation by ACWE.

\section{CONCLUSIONS AND FURTHER WORK}

We have described how new approaches to shape extraction and to low-level processing can be achieved by using physical analogies. Many of the advantages of the new approaches are demonstrated by the results that can be provided. Gravitational force has demonstrated capability for filtering, whilst leading to feature extraction for biometrics. Our new shape extraction approaches evolve from initialisation to a target contour, guided by chosen image properties. Water flow has been demonstrated to good effect when determining shapes in images. Heat has been demonstrated capable of being used as a basis for smooth shape extraction. The new approaches to shape extraction have also proved much faster than a stock comparison technique.

Naturally, the performance of any technique depends on the parameters used to control its operation. It is much easier to explore the effects of parameterisation when the computational basis is simpler, and advantage not enjoyed by the more advanced techniques for determining arbitrary shapes. The analogies used here have implemented basic analysis only and this lends itself to faster execution speed and a simpler choice for parameters. One advantage of the use of analogies is that extensions can be easily made to suit a chosen application. We anticipate that a more routine interface can be made to these new techniques and we are currently working to provide these new interfaces to enable wider migration of these new techniques.

\section{REFERENCES}

[1] Nixon, M. S., and Aguado, A. Image Processing \& Feature Extraction, Academic Press/ Elsevier, $2^{\text {nd }}$ Ed. 2007

[2] Perona, P., and Malik, J., Scale-Space and Edge Detection using Anisotropic Diffusion, IEEE Trans. on PAMI, 17(7), pp 629-639, 1990 
[3] Cremers, D., Tischhäuser, F., Weickert, J., and Schnörr, C., Diffusion Snakes: Introducing Statistical Shape Knowledge into the Mumford-Shah Functional, International Journal of Computer Vision, 50(3), pp 295-313, 2002

[4] Cremers, D., Rousson, M., and Deriche, R., A Review of Statistical Approaches to Level Set Segmentation: Integrating Color, Texture, Motion and Shape, International Journal of Computer Vision, 72(2), pp 195-215, 2007

[5] Chan, T. F., and Vese, L. A., Active Contours Without Edges, IEEE Trans. on IP, 10(2), pp 266-277, 2001

[6] Xie X., and Mirmehdi, M., Magnetostatic Field for the Active Contour Model: A Study in Convergence, in Proceedings of the 17th British Machine Vision Conference, pp 127-136, BMVA press, September 2006

[7] Jalba, A., Wilkinson, M., and Roerdink, J., CPM: A Deformable Model for Shape Recovery and Segmentation based on Charged Particles, IEEE Trans. PAMI, 26, pp 1320-1335, 2004

[8] Vincent, L., and Soille, P., Watersheds in Digital Space: an Efficient Algorithm based on Immersion Simulations. IEEE Trans. PAMI , 13, pp 583-598, 1991

[9] Bleau, A., and Leon, L. J., Watershed-based Segmentation and Region Merging. Computer Vision and Image Understanding, 77, pp 317-370, 2000

[10] Direkoğlu, C., and Nixon, M.S., Low Level Moving-Feature Extraction via Heat Flow Analogy. Proc. International Symposium on Visual Computing (ISVC 2006). LNCS, vol. 4291, pp 243-252, 2006

[11] Acton, S. T., Bovik, A. C., and Crawford, M. M., Anisotropic Diffusion Pyramids for Image Segmentation. Proc. ICIP, 1994

[12] Manay, S., Yezzi, A., Anti-Geometric Diffusion for Adaptive Thresholding and Fast Segmentation. IEEE Trans. on IP, 12(11), 2003

[13] Hurley, D. J., Nixon, M. S., and Carter, J. N., Force Field Energy Functionals for Image Feature Extraction, Image and Vision Computing, 20, pp 311-317, 2002

[14] Hurley, D. J., Nixon, M. S., and Carter, J. N., Force Field Feature Extraction for Ear Biometrics, Computer Vision and Image Understanding, 98(3), pp 491-512, 2005

[15] Liu, X. U., and Nixon, M. S., Water Flow Based Complex Feature Extraction. Proc. Advanced Concepts for Intelligent Vision Systems 2006, Lecture Notes in Computer Science, 4179, pp 833-845, Antwerp, Belgium, 2006

[16] Liu, X. U., and Nixon, M. S., Image and Volume Segmentation by Water Flow, Proc. International Symposium on Visual Computing (ISVC 2007), Nevada, pp62-74, 2007

[17] Direkoğlu, C., and Nixon, M. S., Shape Extraction via Heat Flow Analogy. Proc. International Conference on Advanced Concepts for Intelligent Vision Systems (ACIVS 2007), LNCS 4678, 2007

[18] Direkoğlu, C., and Nixon, M. S., Shape Classification using Multiscale Fourier-based Description in 2-D Space, Proc. IEEE International Conference on Signal Processing 2008 